\title{
Carbon and oxygen stars evolution in post-AGB phase
}

\author{
N. Siódmiak ${ }^{1,2}$, R. Szczerba ${ }^{2}$, M. Meixner ${ }^{1}$, and G. Stasińska ${ }^{3}$ \\ ${ }^{1}$ Space Telescope Science Institute, 3700 San Martin Drive, Baltimore, MD 21218, USA \\ ${ }^{2}$ N. Copernicus Astronomical Center, Rabianska 8, 87-100 Torun, Poland \\ ${ }^{3}$ LUTH, Observatoire de Meudon, 5 place Jules Janssen, 92195 Meudon Cedex, France
}

\begin{abstract}
Despite many studies, the post-AGB phase is still not well understood. To make progress in this field, we searched for information about proto-planetary nebulae and built a catalogue of post-AGB objects. Based on collected data we were able to trace the evolution of stars in their late stages making a distinction between carbon and oxygen-rich objects. We focused our attention on spectral features seen in ISO data and clues for AGB nucleosynthesis. Together with the newest HST images of post-AGB objects we can study correlations between morphological types and chemical and physical properties of stars to improve our understanding of stellar evolution.
\end{abstract}

Keywords. stars: AGB and post-AGB, stars: abundances, stars: imaging

\section{Catalogue of post-AGB objects}

Following the first approach to a catalogue of post-AGB objects (Szczerba et al. 2001) we reviewed available literature and databases to find new candidates and to collect stellar parameters and chemical composition of stars. The present version of the catalogue (Szczerba et al., in preparation) contains about 330 objects. We were able to examine the behavior of objects according to their dominant chemistry: oxygen or carbon-rich. Comparison with theoretical evolutionary paths in the $\left(\log T_{\text {eff }}, \log g\right)$ plane allowed us to estimate the masses of stars. A significant fraction of post-AGB objects are low mass objects $\left(M \sim 0.55 M_{\odot}\right.$ and below) that probably will never become planetary nebulae.

\section{AGB nucleosynthesis}

The efficiency of nucleosynthesis depends on both the metallicity and mass of the star. Since Fe suffers from dust-gas separation (e.g. Mathis \& Lamers 1992) it cannot be used as an indicator of initial chemical composition in post-AGB stars. S and Zn seem to be better indicators for our study because their abundances are (almost) not modified during stellar evolution. Our main findings are as follows (Stasińska et al. 2006): 1) 3rd dredgeup is more efficient at low metallicity, which is compatible with theoretical models (e.g. Marigo 2001), 2) the vast majority of post-AGB objects have experienced 3rd dredgeup, which results in $(\mathrm{C}+\mathrm{N}+\mathrm{O}) / \mathrm{S}$ larger than in the Sun; it seems also that the mass distributions of $\mathrm{C}$ - and O-stars do not differ much with the exception of the lowest mass objects which are dominated by oxygen stars, 3) there is a clear anticorrelation between $\mathrm{N}$ and $\mathrm{O}$ for helium stars that can be interpreted as a production of $\mathrm{N}$ at the expense of $\mathrm{O}$ during the ON cycle being brought to the surface by 2nd dredge-up. 


\section{Infrared spectral features}

There are 83 ISO SWS01 spectra for 65 post-AGB objects from our catalogue. We analyzed characteristic spectral features, especially the $21 \mu \mathrm{m}$ feature. There are only 12 known post-AGB objects which exhibit this feature and they are carbon- and s-processelements-rich. The origin of the $21 \mu \mathrm{m}$ feature is not known and all hitherto proposed band carriers were discarded (e.g. Andersen et al. 2005). We found correlations between the equivalent width (EW) of the $21 \mu \mathrm{m}$ feature and both metallicity $([\mathrm{Fe} / \mathrm{H}]$, Fig. 1$)$ and effective temperature $T_{\text {eff }}$. The correlation between [hs/ls] and $[\mathrm{s} / \mathrm{Fe}]$, as well as between $[\mathrm{hs} / \mathrm{ls}]$ and $[\mathrm{Fe} / \mathrm{H}]$ found by Van Winckel \& Reyniers (2000) and our results may suggest that the carrier of the $21 \mu \mathrm{m}$ feature is likely to contain heavy s-process element(s).

\section{HST images}

We have new images of post-AGB objects from our HST snapshot survey. For the first time we can look at IRAS 15553-5230 in the optical and show its accurate position. Fig. 2 shows 2 images of IRAS 15553-5230 in the K band (from 2-m telescope at Siding Springs Observatory, 2002, by courtesy of G. Van de Steene) and I band (HST, 2005), both covering the same area. Having more images of post-AGB objects we can connect their morphological types with other properties of stars. Similar studies were done in the past already, revealing 2 groups of objects with quite distinct physical parameters (Ueta et al. 2000). Based on those results and new data from the HST survey we hope to improve our understanding of the mechanism that breaks the symmetry of AGB mass loss.

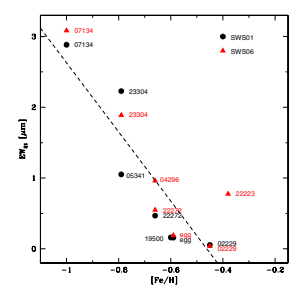

Figure 1. $\mathrm{EW}_{21}$ vs. $[\mathrm{Fe} / \mathrm{H}]$.

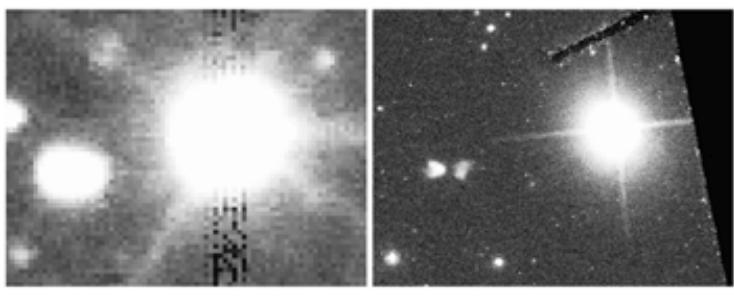

Figure 2. K and I images of IRAS 15553-5230.

\section{Acknowledgements}

This work has been supported by grant 2.P03D.017.25 of the Polish State Committee for Scientific Research, by NASA NAG5-12595, by STScI-G0-10627.01 and by the LEA Astro-PF.

\section{References}

Andersen, A.C., Posch, T., \& Mutschke, H. 2005, in: A. Wilson (ed.), Proceedings of the dusty and molecular universe: a prelude to Herschel and ALMA ESA SP-577, p. 447

Marigo, P. 2001, A\&A 370, 194

Mathis, J.S. \& Lamers, H.J.G.L.M 1992, A\&3A Letters 259, L39

Stasińska, G., Szczerba, R., Schmidt, M., \& Siódmiak, N. 2006, A 6 A 450, 701

Szczerba, R., Górny, S.K., \& Zalfresso-Jundzillo M. 2001, in: R. Szczerba \& S.K. Górny (ed.), Post-AGB Objects as a Phase of Stellar Evolution, ASSL, vol. 265, p. 13

Ueta, T., Meixner, M., \& Bobrowsky, M. 2000, ApJ 528, 861

Van Winckel, H. \& Reyniers M. 2000, A\&A 354, 135 